\title{
Characteristic Functions and Their Empirical Counterparts: Geometrical Interpretations and Applications to Statistical Inference
}

\author{
T. W. EPPS*
}

\begin{abstract}
The value of a characteristic function of a random variable $X$ at some real number $t$ is the center of mass of the distribution of $t X$ wrapped around the unit circle in the complex plane. This geometrical representation is used to illustrate how various properties of frequency functions and characteristic functions correspond and to illuminate the role of empirical characteristic functions in statistical inference.
\end{abstract}

KEY WORDS: Empirical characteristic function; Fourier transform; Wrapped-around distribution.

\section{INTRODUCTION}

In view of the one-to-one correspondence between frequency functions and characteristic functions, their Fourier transforms, models of distributions can be represented equivalently by either function. In practice, the frequency function is the usual representation, because it is the more intuitive concept and because the standard tools of statistical inference rely on the likelihood function or the empirical distribution function. On the other hand, the characteristic function is the canonical representation of some useful distributions whose frequency functions cannot be expressed in closed form. The example most often encountered in applied work is the "stable" laws, the class of limit distributions of sums of iid random variables, which are often used to model such "noisy" processes as common-stock returns. The difficulty of applying likelihood methods to these models led to the use of procedures based on the empirical characteristic function. For example, Paulson, Holcomb, and Leitch (1975) estimated parameters of the stable distributions by minimizing a measure of the total distance between the empirical and stable cfs. Koutrouvelis (1980) devised a regression technique for the same problem, which exploits the particular functional form of the stable cf. Bryant and Paulson (1983) and others have described procedures for estimating parameters of mixture distributions, whose likelihoods - often unbounded - also make traditional inference difficult. These and other papers, notably those of Heathcote (1972, 1977), Feuerverger and Mureika (1977), and Csörgõ (1981), stimulated the wider application of cf methods in inference.

A particularly fertile application of the empirical cf

${ }^{*}$ T. W. Epps is Professor, Department of Economics, University of Virginia, Charlottesville, VA 22901. The author thanks an associate editor and two referees for comments that greatly improved the article. is to tests of various nonparametric hypotheses, such as goodness of fit, K-sample homogeneity, symmetry, and independence. Csörgõ (1984) surveys techniques in these areas that are appropriate for random samples. Epps (1987, 1988) has described applications in the non-iid case also, for testing that time series are Gaussian or stationary. The usefulness of cf methods has been demonstrated even in areas to which frequency-based tools are well adapted, such as testing for normality (Baringhaus and Henze 1988; Epps and Pulley 1983; Hall and Welsh 1983), for two-sample homogeneity (Epps and Singleton 1986), and for exponentiality (Epps and Pulley 1986). In many cases the cf methods are extremely easy to use, relying on statistics that are readily computed and interpreted. In some situations, such as testing for univariate and multivariate normality, it has been argued (Baringhaus, Danschke, and Henze 1989) that they are the best methods available. Still, it is safe to say that these new techniques are not yet widely used by applied statisticians.

A factor that has limited the use of cf's in inference is that statisticians are accustomed to thinking of models in terms of frequency functions. Properties such as location, dispersion, skewness, and tail thickness have clear geometrical interpretations in this context. By contrast, although the mathematics behind many of the properties of cf's is straightforward-Lukacs (1970) is a definitive source - it is not easy to acquire an intuitive understanding. This article uses the geometrical representation of cf's to illuminate some of their properties and to further motivate their use in applied statistical inference. Section 2 develops the geometrical representation of the $\mathrm{cf}$ as the center of mass of a distribution wrapped around the unit circle. Several of the elementary features of cf's are then explained in terms of this paradigm by means of illustrations. Section 3 describes the empirical cf and its applications in inference.

\section{THE GEOMETRICAL REPRESENTATION OF cf's}

For a random variable $X$ with distribution function $F$, the cf, evaluated at arbitrary real $t$, is the FourierStieltjes transform

$$
C(t)=\int e^{i t x} \cdot d F(x) .
$$

In the absolutely continuous and purely discrete cases, $C(t)$ is the ordinary Fourier transform of the density function $\int e^{i t x} \cdot f(x) d x$, or of the probability function, $\sum e^{i t x} \cdot f(x)$.

The transformation $e^{i t X} \equiv \cos (t X)+i \cdot \sin (t X)$ takes 


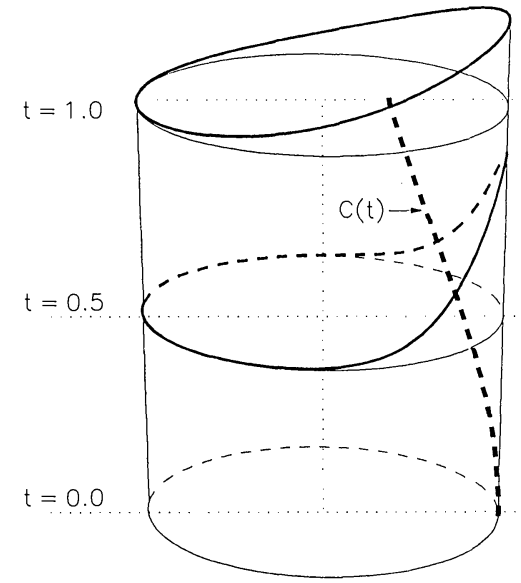

Figure 1. Wrapped-Around Distributions of $t X, X \sim N(0,1)$, at $t=.0, .5,1.0$ and cf of $N(0,1)$ on $[0,1]$.

realizations of $X$ from the real line to the perimeter of the unit circle in the complex plane. If a discrete variable $X$ has positive probability mass at $x$, then in the distribution of the complex variable $e^{i t X}$ this mass appears at a point in the complex plane at angle $t x$ from the real axis. For $t \neq 0$ the total mass at this point is the sum of masses at all points $x+2 \pi j / t, j=0,1,2$, $\ldots$. Thus in the discrete case the probability function of $e^{i t X}$ is that of $t X$ wrapped around the unit circle. The same view applies in the continuous case also, upon substituting the concept of density for mass. Figure 1 depicts the wrapped-around distributions of $t X$ for three values of $t$ when $X$ is $N(0,1)$. In the figure the values $t=.0, t=.5$, and $t=1.0$ correspond to the base, center, and top of the cylinder. The circular contour at each elevation represents the complex plane, with the real axis appearing as the horizontal dotted line. The right-most point on each circle corresponds to $(1,0)$. Drawn on the side of the cylinder at each elevation, and centered on $(1,0)$, is a wrapped-around normal curve with scale $t$. The distribution corresponding to $t=0$ is of course degenerate, with unit mass at $(1,0)$.

Since $C(t)=E\left(e^{i t X}\right)$, the cf at $t \in \mathbf{R}$ is merely the center of mass of the wrapped-around distribution of $t X$. The Cauchy distribution affords a familiar example of the fact that the distribution of $X$ itself sometimes has no definite center of mass. Since expectations of unbounded functions such as $e^{t X}$ often fail to exist, many random variables do not possess moment-generating functions (mgf's). On the other hand, as the center of mass of the wrapped-around distribution of $t X, C(t)$ always exists, lying on or within the unit circle in the complex plane. It lies on the boundary of the circle, in which case $|C(t)|=1$, iff the wrapped-around distribution is degenerate, with all the probability mass concentrated at a single point. This is true, of course, if the distribution of $t X$ itself is degenerate, as it always is at $t=0$; but we shall see later that the distribution of $e^{i t X}$ can be degenerate even when that of $t X$ is not. A nondegenerate wrapped-around distribution clearly must have center of mass within the unit circle. Thus, taking the domain of $C(\cdot)$ to be the entire real line, we have the geometrical representation of the cf as a curve confined to an infinitely long circular cylinderhenceforth, the "unit" cylinder-and having the value $(1,0)$ at $t=0$. The bold dashed line in Figure 1 represents the standard-normal $\mathrm{cf}$ on the set $[0,1]$, and Figure 2 depicts that cf and two others on $[0,2]$.

As the center of mass of the wrapped-around distribution, the cf corresponding to a frequency function that is symmetric about the origin clearly lies always on the real axis of the unit cylinder. Figure 1 illustrates this fact. If $X$ has such a symmetric distribution and $Y=$ $X+\mu$, then the distribution of $e^{i t Y}$ is merely that of $e^{i t X}$ rotated by angle $t \mu$. As $t$ varies, the center of mass of the distribution of $e^{i t Y}$ thus has a cyclic component that causes it to rotate about the center of the cylinder. The distance from the center, $\left|C_{Y}(t)\right|$, always equals $\left|C_{X}(t)\right|$. The cf's of the distributions $N(0,1)$ and $N(5$, $1)$ in Figure 2 illustrate this behavior. The third curve in Figure 2 depicts the of of the degenerate random variable $X=5$, which always resides on the surface of the unit cylinder. At any $t$ the cf of $N(5,1)$ is the product of the cf's of $N(0,1)$ and of the degenerate variable. Since $|C(t)|=1$ only if the wrapped-around distribution of $t X$ is degenerate, a cf can lie on the surface of the unit cylinder for all values of $t$ only if the distribution of $X$ itself is degenerate. Because $C(0)=(1,0)$ always, a cf can be constant only if $C(t)=(1,0)$, which requires $P\{X=0\}=1$.

Comparing the wrapped-around distributions of $t X$ and $-t X(t \neq 0)$, one is seen to be a mirror image of the other about the real axis. Since the real parts of their centers of mass are the same and the imaginary
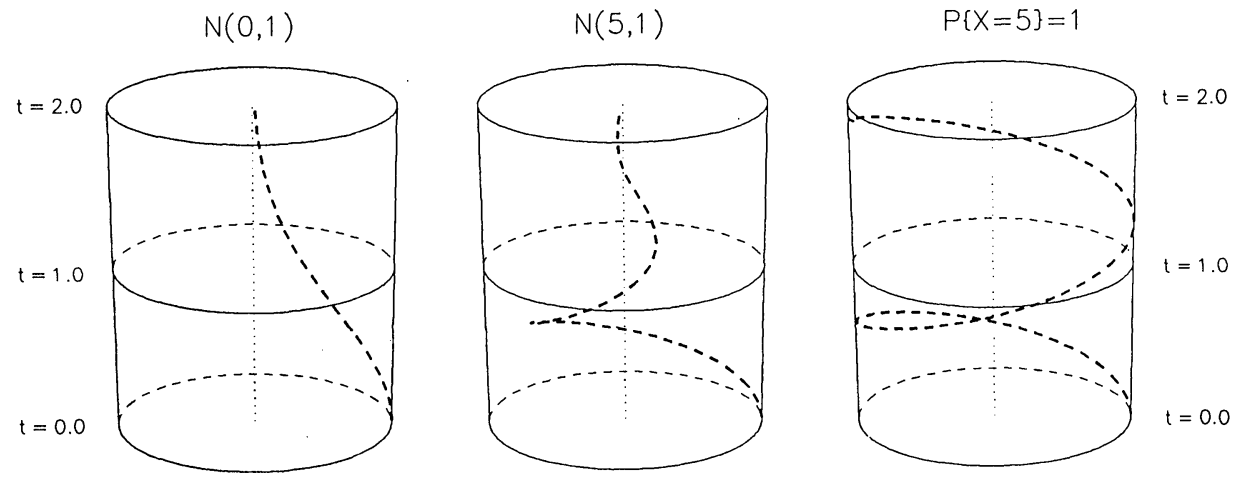

Figure 2. cf's of $N(0,1), N(5,1)$, and a Degenerate Distribution on [0, 2]. 
parts are equal but of opposite sign, $C(-t)$ is the complex conjugate of $C(t)$; accordingly, the restriction of $C(t)$ to $[0, \infty)$ fully represents the function.

We have seen that the cf of a degenerate random variable always resides on the surface of the unit cylinder. Consider the behavior of cf's of absolutely continuous random variables and of discrete variables whose distributions are supported on a lattice. In the continuous case, as $t$ increases from zero the wrapped-around distribution of $t X$ approaches that of a uniform distribution with density $1 /(2 \pi)$ on the perimeter of the unit circle. (To visualize this approach to uniformity, it may help to think of wrapping the distribution of $X$ itself about a circle of radius $1 / t$, and letting $t \rightarrow \infty$.) Since the center of mass of this uniform distribution is at the origin, one can see that for an absolutely continuous random variable (rv) $|C(t)| \rightarrow 0$ as $t \rightarrow \infty$. On the other hand, the presence of an atom of mass at any point $x$ would contribute to the cf a cyclic component, $e^{i t x}$. $P\{X=x\}$, that would keep it from damping out as $t \rightarrow \infty$.

Turning to lattice distributions, all of whose probability mass is in the form of atoms on a subset of a collection of equally spaced points, consider first the simple case of a Bernoulli variate, with $P\{X=0\}=$ $p=1-P\{X=1\}$. The distribution of $e^{i t X}$ has mass $p$ at $(1,0)$ and mass $1-p$ at $e^{i t}$. As $t$ increases from zero, the mass at $e^{i t}$ moves around the unit circle until, at $t=2 \pi$, all the mass comes to reside at $(1,0)$. Thus, the $\mathrm{cf}$ is periodic, with period $2 \pi$. This illustrates the fact that the distribution of $e^{i t X}$ can be degenerate even when that of $t X$ itself is not. More generally, if $X$ has a distribution supported on two or more points of the set $\{a, a \pm d, a \pm 2 d, \ldots\}, d>0$, then adjacent mass points of the wrapped-around distribution of $t X$ are points on the unit circle separated by angle $t d$. For values of $t$ that are integral multiples of $2 \pi / d$ all the mass is concentrated at the point $e^{i t a}$. Thus, the modulus of the cf of any distribution supported on a lattice is periodic, with period $2 \pi / d$. If the lattice representation allows $a=0$, as in the Bernoulli case-indeed, whenever ald is rational - then $C(t)$ itself is strictly periodic.

Figure 3 depicts the periodic behavior of the binomial

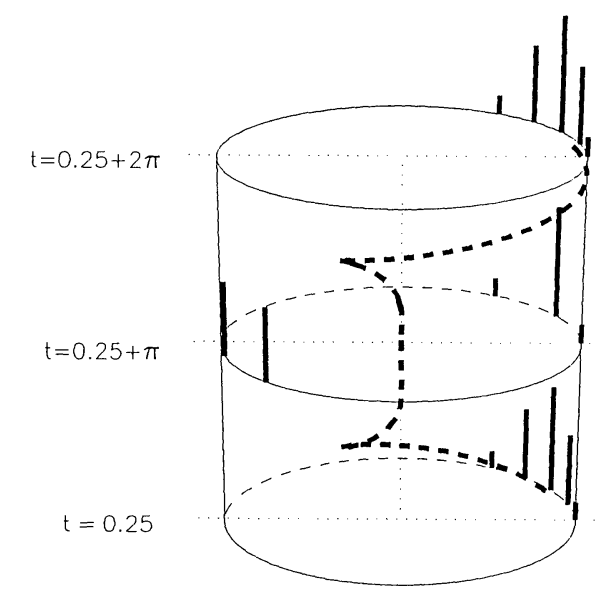

Figure 3. Periodic Behavior of the Binomial $(4, .5) \mathrm{cf}$.

Mixture:

Laplace $(0, .25)$

Laplace $(-1,25)$,

Laploce $(1, .25)$
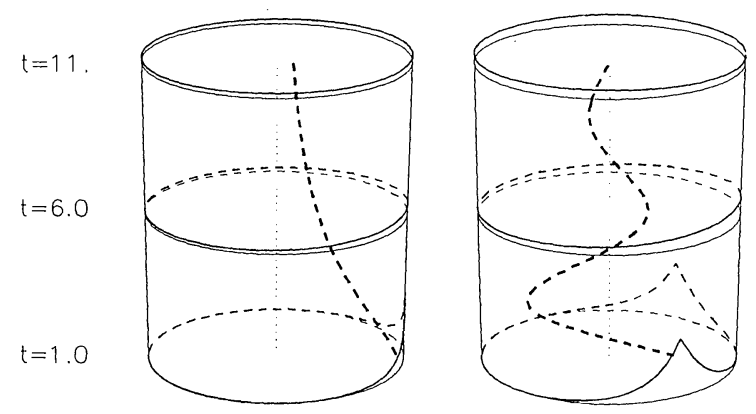

Figure 4. Oscillatory Behavior of cf's of Bimodal Distributions.

$(4, .5) \mathrm{cf}(a=0, d=1)$. At the bottom level, $t=.25$, the five probability masses, $\left\{\left(\begin{array}{l}4 \\ x\end{array}\right) / 16\right\}_{x=0}^{4}$, are separated by the angle .25 . As $t$ increases to $.25+\pi$, the masses corresponding to $x=1$ and $x=3$ rotate through angles $\pi$ and $3 \pi$, to appear just opposite their first positions, while the masses at 2 and 4 move around the circle once and twice to their original places. At the top level, $t=$ $.25+2 \pi$, all reoccupy the starting positions.

Just as the of of a distribution with atoms never damps out, the degree of oscillatory behavior of the cf of an absolutely continuous distribution depends on the smoothness of the pdf. For example, cf's of unimodal distributions are typically better behaved than those of bimodal distributions. When the distribution of $t X$ is bimodal, the center of mass of the wrapped-around distribution oscillates as $t$ increases, the modes first spreading apart, achieving at least partial balance, spreading again, and eventually overlapping. The process is depicted in Figure 4. On the left are wrappedaround distributions and $\mathrm{cf}$ of the Laplace with mean zero and scale .25 , denoted $L(0, .25)$. The right figure pertains to an equal mixture of $L(-1, .25)$ and $L(1$, .25). By comparing distributions with real-valued cf'shere, $\left(1+t^{2} / 16\right)^{-1}$ and $\cos (t) \cdot\left(1+t^{2} / 16\right)^{-1}$ - we focus on the effect of bimodality per se. The sign of the cf of the mixture alternates as $t$ increases and the relative positions of the modes change. The distance between the modes governs the frequency of oscillation, and their sharpness governs the amplitude. The oscillation ultimately dies out as the wrapped-round distribution nears uniformity.

It is well-known that the existence of the mgf implies the existence of all moments and that these can be found by differentiating the mgf at the origin. The cf generates moments in much the same way; in particular, $E\left(X^{k}\right)=$ $\left.i^{-k} \cdot C^{(k)}(t)\right|_{t=0}$. Evidently, the thinness of a distribution's tails, which governs the existence of moments, is connected with the differentiability of $C(t)$ at $t=0$. A geometrical interpretation of this feature is available. Figure 5 depicts wrapped-around distributions and the cf of $X \sim L(5,1)$ and of $Y \sim$ Cauchy(median 5.0, scale 1.0). At $t=0$, both wrapped-around distributions are, of course, concentrated at $(1,0)$. As $|t|$ increases, the 

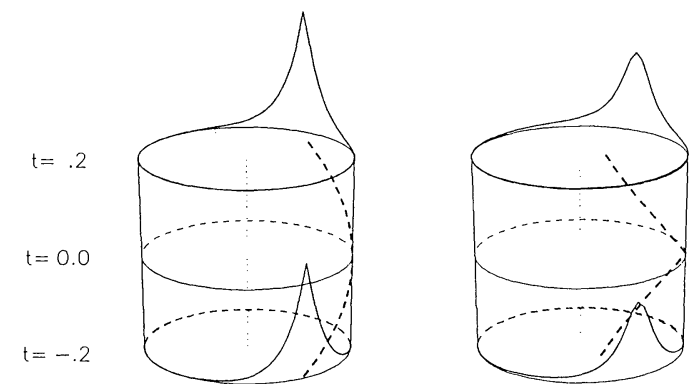

Figure 5. Greater Smoothness at $t=0$ of cf's of Thin-Tailed Distributions.

Cauchy's thick tails cause the center of mass of the distribution of $e^{i t Y}$ to move so sharply toward the origin that $C_{Y}(t)=e^{5 i t-|t|}$ fails to be differentiable at $t=0$. On the other hand, the $\mathrm{cf}$ of the thin-tailed Laplace distribution, $C_{X}(t)=e^{5 i t} /\left(1+t^{2}\right)$, is very smooth.

The Laplace and Cauchy distributions happen to be natural conjugates, in the sense that the modulus of the cf of each has the same form as the pdf of the otherspecifically, $f_{X}(t) \propto\left|C_{Y}(t)\right|$ and $\left.f_{Y}(t) \propto \mid C_{X}(t)\right]$. This special conjugate relation allows Figure 5 to illustrate a general form of symmetry between cf's and frequency functions, arising from their relationship as Fouriertransform pairs: Just as smoothness of the $\mathrm{cf}$ at $t=0$ is associated with thin tails in the distribution, smoothness of the pdf at $x=0$ corresponds to a thin-tailed cf.

An essential property of cf's is their one-to-one correspondence with distribution functions. (Indeed, it is this uniqueness that makes them "characteristic.") It is not at all uncommon for distinct distributions $F_{X}$ and $F_{Y}$ to have cf's that coincide at certain nonzero values of $t$. (Of course, they invariably coincide at $t=0$.) For example, the standard-normal and Cauchy cf's are equal at $t= \pm 2$, and we have seen that all distributions supported on the nonnegative integers coincide at intervals of $2 \pi$. On the other hand, the uniqueness theorem (Lukacs 1970, pp. 28-29) rules out the possibility that cf's of distinct distributions could coincide for all $t$. Let us see how the geometry of cf's makes this intuitively plausible. The difference between two cf's, $C_{X}(t)-C_{Y}(t)=\int \exp (i t x) \cdot d F_{X}(x)-\int \exp (i t y) \cdot$ $d F_{Y}(y)=\int \exp (i t s) \cdot d\left[F_{X}(s)-F_{Y}(s)\right]$, can be viewed as the center of mass of an improper wrapped-around distribution with zero net mass but (signed) mass $d\left[F_{X}(s)-F_{Y}(s)\right]$ on the arc from $\exp (i t s)$ to $\exp [i t(s+$ $d s)$ ]. For example, with discrete $X$ and $Y$ there will be "masses" $f_{X}(s)-f_{Y}(s)$ and $f_{X}\left(s^{\prime}\right)-f_{Y}\left(s^{\prime}\right)$ on the unit circle at angles $t s$ and $t s^{\prime}$ from the real axis, and as $t$ increases these will move around the unit circle at different rates. The condition $C_{X}-C_{Y}=(0,0)$ requires this improper wrapped-around distribution always to have center of mass at the origin. Intuitively, this seems to rule out any nonuniformity in $d\left[F_{X}(s)-F_{Y}(s)\right]$, for as $t$ changes the relative positions of local irregularities in the wrapped-around distribution would change - and thereby move the center of mass. But the improper wrapped-around distribution, having zero net mass, could be constant on the unit circle only by being uniformly zero. This implies that $F_{X} \equiv F_{Y}$.

Although we do not treat them here, we note that certain other features of cf's, such as the form taken by cf's of mixture distributions and of convolutions, also have geometrical interpretations when the cf's are viewed as centers of mass of wrapped-around distributions. As for mgf's, the cf of a sum of independent random variables is the product of their cf's, and the cf of a mixture is a convex linear combination of the individual cf's.

\section{THE EMPIRICAL cf}

If $F_{n}$ is the empirical distribution function (df) corresponding to a sample, $\left\{X_{j}\right\}_{j=1}^{n}$, from a population with distribution $F$, the empirical cf is

$$
C_{n}(t)=\int e^{i t x} \cdot d F_{n}(x) \equiv n^{-1} \sum_{j=1}^{n} \exp \left(i t X_{j}\right) .
$$

As the center of mass of the wrapped-around empirical distribution of $t X, C_{n}(t)$ behaves like the cf of a discrete rv. When $F$ is absolutely continuous, then $F_{n}$ is a.s. not supported on a lattice, so that $C_{n}$ is not strictly, but almost periodic (Feuerverger and Mureika 1977). (In practice there is strict periodicity even in the continuous case because of rounding.) In any case, $C_{n}(\cdot)$ is a complex-valued stochastic process whose realizations are confined to the unit cylinder. Empirical cf's corresponding to samples of size $n=10$ and $n=100$ from a standard-normal population are illustrated in Figure 6. Note the apparent tendency for $C_{n}$ to converge to $C$ as $n$ increases.

In fact, when $\left\{X_{j}\right\}_{j=1}^{n}$ are iid, many of the large-sample properties of $C_{n}$ follow rigorously from the almost-sure uniform convergence of $F_{n}$ to $F$. Feuerverger and $\mathrm{Mu}$ reika (1977) prove the a.s. uniform convergence of $C_{n}$ to $C$ on any finite interval-on $(-\infty, \infty)$ if $F$ is a step function; and Marcus (1981) proves that when $F$ satisfies a weak tail condition the normalized process $\sqrt{n}$. $\left(C_{n}-C\right)$ converges weakly to a zero-mean, complex Gaussian process whose covariance function depends on $C$. Epps (1987) extends the latter result to the case

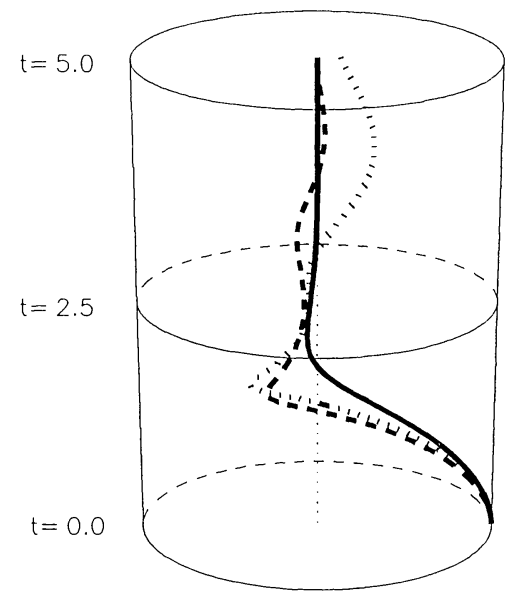

Figure 6. cf and Two ecf's From $N(1,1)$ Population, Sample Sizes 10 and 100. $\cdots, C_{n}(t), n=10 ;---, C_{n}(t), n=100 ;-, C(t)$. 
in which the sample data are themselves the realization of a discrete-time, stationary, Gaussian process - a result which opens up applications of the empirical $\mathrm{cf}$ in time-series analysis. Although these results are quite deep, the weaker, finite-dimensional versions of the theorems are obvious, at least in the iid case, from the geometrical interpretation of $C_{n}$ as the center of mass of the empirical distribution of $\left\{\exp \left(i t X_{j}\right)\right\}_{j=1}^{n}$. These asymptotic properties form the basis for applications of $C_{n}$ in statistical inference.

Many of these applications use one of the following types of functionals of the distance between the cf's of the sample and the model:

$$
\begin{gathered}
\int D\left\{C_{n}(t), C(t)\right\} \cdot d W(t), \\
\int D\left\{C_{n}(t), C(t)\right\} \cdot D\left\{C_{n}(s), C(s)\right\} \cdot d W(s, t),
\end{gathered}
$$

where $D\{\cdot, \cdot\}$ measures distance and $W$ is a weighting function. For example, taking $D\left\{C_{n}, C\right\}=\left|C_{n}-C\right|^{2}$ and $W(t)=\int_{-\infty}^{t} \exp \left(-\alpha u^{2}\right) d u, \alpha>0$, Paulson et al. (1975) and Bryant and Paulson (1983) estimated parameters of the stable laws and mixtures of normals by minimizing $\int\left|C_{n}(t)-C(t)\right|^{2} \cdot \exp \left(-\alpha t^{2}\right) \cdot d t$. Heathcote (1977) showed that such "integrated squared-error" estimators are usually consistent and related their asymptotic relative efficiency to the choice of $W$. Epps and Pulley (1983) and Baringhaus and Henze (1988) used similar integrated-distance statistics in testing univariate and multivariate normality. Using $D\left\{C_{n}, C\right\}=C_{n}-C$ and a certain complex-valued $W$, Epps and Pulley (1986) obtained from (1) a simple and powerful test for exponentiality. Feuerverger and McDunnough (1981a, b) and Koutrouvelis and Kellermeier (1981) developed methods of estimation and tests of fit based on quadratic forms in differences $\left\{C_{n}\left(t_{i}\right)-C\left(t_{i}\right)\right\}_{i=1}^{k}$ on a finite set of points. Functionals like (2) can take such forms if $W(s, t)$ is a step function. The efficiency of minimumdistance estimators of this type can be high if $W(s, t)$ and the $\left\{t_{i}\right\}$ are appropriately chosen.

An example of testing univariate normality will illustrate these distance methods. The data $\left\{x_{j}\right\}_{j=1}^{10} \equiv$ $\{-8.62,-2.88,-1.26,-1.03,-.62, .13, .47, .70$, $1.28,1.79\}$ are a sample of 10 from a standard Laplace distribution, ordered just for display. They were generated by mixing standard exponential variates, obtained via the inverse probability integral transform with the RAN1 generator in Press, Flannery, Teukolsky, and Vetterling (1989), initial seed -4. Figure 7 compares the empirical df of the standardized data, $\left\{y_{j} \equiv\left(x_{j}-\right.\right.$ $\bar{x}) / s\}_{j=1}^{10}$, with the standard-normal df. The outlier in the left tail is prominent. The solid curve in Figure 8 shows the squared modulus of the difference between the empirical and normal cf's weighted by $d W(t)=(2 \pi)^{-1 / 2}$. $\exp \left(-t^{2} / 2\right)$. The large peak around $|t|=1.5$, suggesting poor fit, is greatly diminished when the outlier is excluded (the dashed curve). The total area under the curve, $\int_{\infty}^{\infty}\left|C_{n}(t)-C(t)\right|^{2} \cdot d W(t)$, which is the statistic used in the Epps-Pulley-Baringhaus-Henze test, turns out to be a simple function of the standardized observations: $n^{-1} \cdot \sum \Sigma \exp \left[-\left(y_{j}-y_{k}\right)^{2} / 2\right]-\sqrt{2}$. $\sum \exp \left(-y_{i}^{2} / 4\right)+n \sqrt{3}$. Large values indicate departure

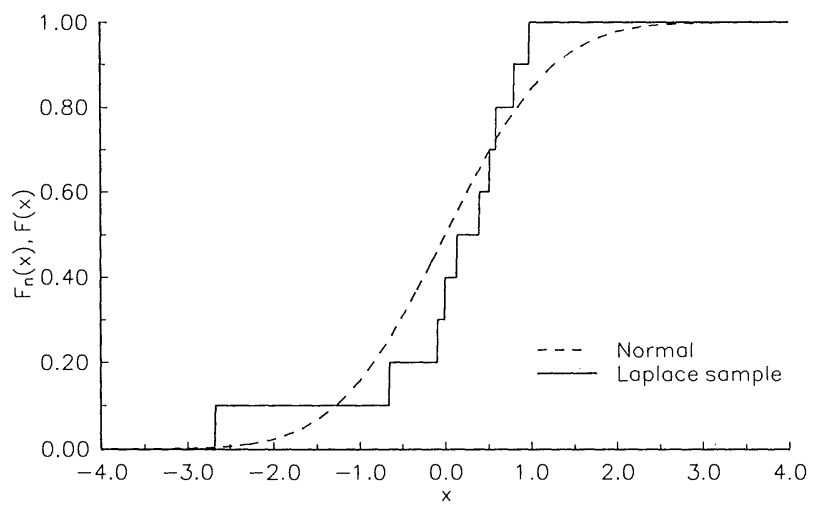

Figure 7. Empirical df of Standardized Laplace and df of Normal, Sample Size 10.

from normality. Its value for the full sample of Laplace data, .582, implies rejection at the .01 level, whereas the value without the outlier, .049, is quite consistent with normality.

The role of $W$ in this and the other tests based on (1) or (2) can be related to the geometrical behavior of the empirical cf. We have seen that the cf of an absolutely continuous distribution approaches $(0,0)$ as $t \rightarrow$ $\infty$, whereas the ecf is periodic. One purpose of $W$, therefore, is just to assure convergence of the integrals. Another is to give the distances $D\left\{C_{n}(t), C(t)\right\}$ high influence at values of $t$ where $C_{n}(t)$ has high precision. It is easy to show that for any real $s, t$

$$
\begin{aligned}
E\left\{\left[C_{n}(s)-C(s)\right] \cdot\left[C_{n}(t)-C(t)\right]\right\} & \\
= & n^{-1} \cdot[C(s+t)-C(s) C(t)] .
\end{aligned}
$$

The quadratic forms used by Feuerverger and McDunnough (1981a), Koutrouvelis and Kellermeier (1981), and Epps (1987) weight vectors of differences between real and imaginary parts of the ecf and cf by the inverse of an appropriate covariance matrix, with elements similar to (3). In this way the test statistic-the minimum of the quadratic form with respect to the parameters is made asymptotically distribution free.

Taking $s=-t$ in (3) shows that the precision of $C_{n}$ declines as $|C(t)|$ departs from unity, a fact that has a ready geometric interpretation. $|C(t)| \simeq 1$ only if the wrapped-around distribution of $t X$ is nearly degenerate.

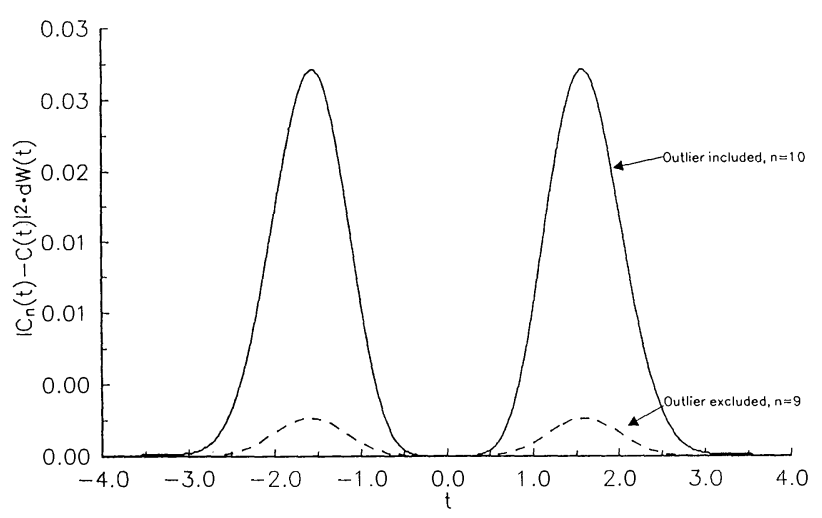

Figure 8. Weighted Squared Modulus, Laplace ecf-Normal cf, With and Without Outlying Observation. 
The probability is then high that the sample points, $\left\{\exp \left(i t X_{j}\right)\right\}_{j=1}^{n}$, will be highly concentrated, so that the mean will have small variance. By contrast, $|C(t)| \simeq 0$ implies that the distribution of $e^{i t X}$ is highly dispersed, so that there will be high variation in $n^{-1} \cdot \Sigma \exp \left(i t X_{j}\right)$ also. In practice, the ecf tends to be most informative near the origin.

\section{SUMMARY}

$C(t)$, the characteristic function of a random variable $X$ evaluated at some real number $t$, has a geometrical interpretation as the center of mass of the distribution of $t X$ wrapped about the unit circle in the complex plane. This interpretation clarifies the elementary properties of cf's and relates them to the properties of frequency functions. The geometrical view also illuminates some of the features of inferential techniques that employ the empirical cf.

[Received July 1991. Revised March 1992.]

\section{REFERENCES}

Baringhaus, L., Danschke, R., and Henze, N. (1989), "Recent and Classical Tests for Normality-a Comparative Study," Communications in Statistics, 18, 363-379.

Baringhaus, L., and Henze, N. (1988), "A Consistent Test for Multivariate Normality Based on the Empirical Characteristic Function, Metrika, 35, 339-348.

Bryant, J. L., and Paulson, A. S. (1983), Estimation of Mixing Proportions via Distance Between Characteristic Functions," Communications in Statistics, Part A-Theory and Methods, 12, 10091029.

Csörgõ, S. (1981), "Limit Behavior of the Empirical Characteristic Function," Annals of Probability, 9, 130-144. - (1984), "Testing by the Empirical Characteristic Function: A Survey," in Asymptotic Statistics 2: Proceedings of the 3rd Prague Symposium on Asymptotic Statistics, eds. P. Mandl and M. Muskova, Amsterdam: Elsevier.
Epps, T. W. (1987), "Testing That a Stationary Time Series is Gaussian," Annals of Statistics, 15, 1683-1698.

(1988), "Testing That a Gaussian Process is Stationary," Annals of Statistics, 16, 1667-1683.

Epps, T. W., and Pulley, L. B. (1983), "A Test for Normality Based on the Empirical Characteristic Function," Biometrika, 70, 723726 .

- (1986), “A Test of Exponentiality vs. Monotone-Hazard Alternatives Derived From the Empirical Characteristic Function, Journal of the Royal Statistical Society, Ser. B, 48, 206-213.

Epps, T. W., and Singleton, K. J. (1986), "An Omnibus Test for the Two-Sample Problem Using the Empirical Characteristic Function," Journal of Statistical Computation and Simulation, 26, 177203.

Feuerverger, A., and McDunnough, P. (1981a), "On the Efficiency of Empirical Characteristic Function Procedures, Journal of the Royal Statistical Society, Ser. B, 43, 20-27.

(1981b), "On Some Fourier Methods for Inference," Journal of the American Statistical Association, 76, 379-387.

Feuerverger, A., and Mureika, R. A. (1977), "The Empirical Characteristic Function and Its Applications, Annals of Statistics, 5, 8897.

Hall, P., and Welsh, A. H. (1983), "A Test for Normality Based on the Empirical Characteristic Function," Biometrika, 70, 485-489.

Heathcote, C. R. (1972), "A Test of Goodness of Fit for Symmetric Random Variables," Australian Journal of Statistics, 14, 172-181.

(1977), "The Integrated Squared Error Estimation of Parameters," Biometrika, 64, 255-264.

Koutrouvelis, I. A. (1980), "Regression-Type Estimation of the Parameters of Stable Laws," Journal of the American Statistical Association, 75, 918-928.

Koutrouvelis, I. A., and Kellermeier, J. (1981), "A Goodness-of-Fit Test Based on the Empirical Characteristic Function When Parameters Must Be Estimated," Journal of the Royal Statistical Society, Ser. B, 43, 173-176.

Lukacs, E. (1970). Characteristic Functions (2nd ed.), London: Griffin.

Marcus, M. B. (1981), "Weak-Convergence of the Empirical Characteristic Function," Annals of Probability, 9, 194-201.

Paulson, A. S., Holcomb, E. W., and Leitch, R. A. (1975), "The Estimation of the Parameters of the Stable Laws," Biometrika, 62, 163-170.

Press, W. H., Flannery, B. P., Teukolsky, S. A., and Vetterling, W. T. (1989), Numerical Recipes (FORTRAN Version), Cambridge: Cambridge Press. 